\title{
Surface tensometry studies on formulations of surfactants with preservatives as a tool for antimicrobial drug protection characterization
}

\author{
Stanislaw Pogorzelski ${ }^{1^{*}}$, Dorota Watrobska-Swietlikowska², Malgorzata Sznitowska ${ }^{2}$ \\ ${ }^{1}$ Institute of Experimental Physics, University of Gdansk, Gdansk, Poland; ${ }^{*}$ Corresponding Author: fizsp@ug.edu.pl \\ ${ }^{2}$ Department of Pharmaceutical Technology, Medical University of Gdansk, Gdansk, Poland
}

Received 17 August 2012; revised 25 September 2012; accepted 8 October 2012

\begin{abstract}
The aim of the work was to quantify possible interactions between surfactants and preservatives, comparing surface properties, in model pharmaceutical formulations. Surface parameters of 2-component surfactant-preservative aquous mixtures were determined with a Wilhelmy plate technique, for the so-called principal surfactants (polysorbate 80, egg lecithin, phosphatidylcholine) and preservatives, which were methylparaben and benzalkonium chloride (BAC). A generalized surface tension vs. surfactant concentration plot signatures, in the presence of preservative at a fixed amount, allowed: the critical micellar concentration (cmc) shift, additive molecules partition from the surface to the bulk, mixed micelles formation concentration, and additive surface removal concentration to be determined in reference to surface activity of the added substance. Methylparaben is a compound of lower (in comparison to BAC) surface activity, lower partitioning coefficient possessing lower energy and concentration of its removal from the surface, that makes it play effectively an antimicrobial protection role in the bulk of pharmaceutical products, as already shown by chemical tests.
\end{abstract}

Keywords: Drug-Preservative Mixtures; Surface Tension; Surface Activity; Interfacial Partitioning; Antimicrobial Drug Protection

\section{INTRODUCTION}

Pharmaceutical products when distributed into multidose containers should be properly preserved against microbial contamination and proliferation during storage under normal conditions and effective use. Particularly, high enough concentration of free preservative agents in water is required to achieve sufficient antimicrobial protection.

In the light of the complexity of the drug-preservative system, it is clear that preservatives may remain more or less available for the contact with microorganisms, depending on the site of their location, i.e., air/water interfacial region, bulk water phase or being incorporated in the liposomal or micellar structures in the complex mixtures. In the previous work [1], preservatives for submitcron emulsions were proposed on the basis of their physicochemical compatibility, and as the most promising parabens were chosen. In these studies an attempt was made to provide a solution to the problem of efficient selection a microbial protection agent, based on physical surface adsorption attributes.

Surface tensiometry studies performed here on model 2-component drug-preservative mixtures allowed to distinguish between the fractions of free preservatives, adsorbed at interfaces or incorporated in the mixed micellar phase in reference to chemical structure, surface activity and mixed micelles formation ability of the particular preservative.

The interfacial sensitive and quantitative parameters: surface adsorption, partitioning of surfactant molecules between surface and bulk phases, additive surface activety and its surface effectiveness $\gamma_{\mathrm{cmc}}$, molecular interfacial area, cmc of mixed micelles formation, and surface removal concentration $c_{\text {rem }}$ were demonstrated to be useful indicators to perform proper preservative selection.

Critical micelle concentration $(\mathrm{cmc})$, mixed micelles formation are determined for chosen 2-component systems using the surface partitioning model based on surface tension versus concentration data [2]. Application of surface tensiometry is supposed to provide a more detailed interpretation on intermolecular interactions, and partitioning coefficients in model mixtures of egg lecithin and methylparaben, as already studied before by chemical analyses [3], that is a key problem in the opti- 
mal drug formulation procedures.

Adsorption of surfactants at A/W (air/water) interface is expressed quantitatively by Gibbs' equation [4]:

$$
\Gamma=\frac{-1}{R T} \frac{\mathrm{d} \gamma}{\mathrm{d} \ln c}
$$

in which $\Gamma$ is the surface excess (adsorption) related to the area per molecule $A_{\text {molec }}=1 / \Gamma \mathrm{N}_{\mathrm{A}} ; \gamma$ the surface tension; c the bulk concentration of surfactant; $\mathrm{R}$ the gas constant; $\mathrm{T}$ is the absolute temperature, and $\mathrm{N}_{\mathrm{A}}$ the Avogadro nu- mber. For ionic surfactants, a denominator in Eq.1 is multiplied by a factor of 2. The slope of $\gamma$ plotted versus $\ln$ concentration is proportional to surface excess $\Gamma$ and further to the molecular area $A_{\text {molec }}$.

Adsorption of amphiphile at the air/water interface leads to $\gamma$ decrease as the total concentration is raised. A point is reached at which the interface becomes saturated with monomers $\left(\Gamma=\Gamma_{\infty}\right.$ at $\left.c=\mathrm{cmc}\right)$. Consequently, $\gamma$ levels off. The concentration range, above such a breaking point at $\gamma(c)$ dependence, is referred as the micelle formation region. The surface activity of a surfactant in the solution $\sigma$ (see Eq.2), is proportional to the partitioning coefficient $\mathrm{K}_{\mathrm{p}}=\Gamma / \mathrm{c}$ of the surfactant molecules between the surface and bulk phases, as results from Eq.1:

$$
-\frac{\mathrm{d} \gamma}{\mathrm{d} c}=\sigma=\Gamma \frac{R T}{c}
$$

Intercomparisons of the surface rheological properties of surface films occurring at interfaces of different surfactant-containing systems requires the partitioning effect of the surfactant molecules between the surface and bulk phases to be estimated [5]. Owing to mass conservation, the total amount of surfactant, $n$ (mol), must be subject to the relation:

$$
n=A \Gamma+V c
$$

involving the interfacial area $A\left(\mathrm{~cm}^{2}\right)$, the surface concentration $\Gamma$ (mol per unit area), the bulk volume $V\left(\mathrm{~cm}^{3}\right)$, and the subphase concentration $c$ (mol per unit volume), respectively.

The adsorption at the interface in a mixture of surfactants results in the formation of a mixed monolayer. The composition of mixed adsorbed films depends on the concentration ratio of the surfactants, the difference in their surface activity and the concentration of electrolytes in the solution. For a surfactant mixture of two-component surfactants, if the components are immiscible or they behave like an ideal mixture, the following relationship is valid [6]:

$$
A_{12}=x_{1} A_{1}+x_{2} A_{2}
$$

where $A_{12}$ is the molecular area in the mixture monolayer at a fixed surface pressure $\pi, A_{1}$ and $A_{2}$ are the molecular areas in the pure component monolayer at the same $\pi$, and $x_{1}$ and $x_{2}$ are the molar or weight fractions of the pure components in the mixture such that $x_{1}+x_{2}=1$.

Positive or negative deviations from Eq.4 i.e., $\Delta A_{\text {molec }}$ $=A_{\text {exp }}-A_{12}$, where $A_{\text {exp }}$ is the experimentally-determined mixed film molecular area, indicates the presence of repulsive or attractive interactions, respectively between the two components in the mixed monolayers [7].

The critical micelle concentration $\mathrm{cmc}$ of a mixture of similarly structured ionic surfactants or nonionic ones can be predicted reasonably well by assuming that ideal solution theory is obeyed in the micellar phase. Eq.4 remains valid where $A_{\text {molec }}$ quantity is substituted with cmc. However, the cmc of mixtures of nonionic and ionic surfactants can be much less than predicted from ideal solution theory [8]. A method of calculating the composition of mixed micelles in equilibrium with monomer of known composition is proposed that needs only cmc data as a function of monomer composition, but is limited to binary surfactant systems [9].

In order to determine the surface activity of such additives, important for surface active surfactant-preservative systems, surface tension measurements of pure components and mixed aqueous solutions, appearing in a wide range of concentrations, from pre-micellar to above $\mathrm{cmc}$ ranges, were performed for model compounds: surfacetants (egg lecithin, phosphatidylcholine and polysorbate 80) and preservatives (BAC and methylparaben) playing a role of microbial preservatives.

It should be pointed out that the studied components of pharmaceutical concern are differentiated in nature. Lecithin is a mixture of phospholipids with phosphatidylcholine as a main component (up to $98 \% \mathrm{w} / \mathrm{w}$ ). Egg or soya lecithin as well as purified phospholipids are used for pharmaceutical purposes as components of liposomes, mixed micelles and submicron emulsions. Lecithin does not dissolve completely in water. Aqueous dispersion of lecithin is a yellowish, non-transparent homogenous liquid, free of undissolved particles, when visually inspectted. Using a laser diffractometer, particles in the range of $0.05-2 \mu \mathrm{m}$ were evidenced. Preliminary electron microscopic observation revealed the presence of structured, aggregated particles [10]. According to other authors many different structures like: liposomes, lamellar or discoidal micelles, nanoparticles or network-like are present in the systems containing phospholipids structures [11-14], and most probably these structures can also be present in aqueous dispersions of lecithin investigated in the present study. BAC and methylparaben are tensioactive compounds and appeared in the studied submicron emulsion as preservatives at concentrations of $0.2-0.05 \mathrm{mg} / \mathrm{g}$ and $1.8 \mathrm{mg} / \mathrm{g}$ (all below $\mathrm{cmc}$ ), respectively [3]. BAC is a cationic surfactant but methylparaben despite its surface activity does not form micelles. Due to complex structures present in the lecithin- 
containing systems, this surfactant in water cannot be considered as simple micellar dispersions, although for the purpose of this article, the observed physical events are discussed referring to micelles in the system.

With the aim to better understand physical events occurring in pharmaceutical preparations containing lecithin it is important to investigate surface potential of lecithin dispersions in water and the effect of other excipients on its value. Since preservatives are common pharmaceutical excipients surface characteristics of lecithin was studied in their presence and this was compared with systems containing classical surfactant, polysorbate 80 , instead of lecithin.

This study proposes a physicochemical characterization methodology as a tool for preservative selection and could be applied to liquids and semi-solids formulations. Possible physicochemical and chemical interactions between preservatives and other formulation components must be predictable and well known. It is noteworthy to mention the importance of understanding these interacttions in order to add supplementary information to microbiological tests.

\section{MATERIALS AND METHODS}

\subsection{Materials}

Surfactant solutions were prepared using purified water (produced in Millipore reverse-osmosis apparatus) with conductivity $0.05 \mu \mathrm{S} / \mathrm{cm}, \mathrm{pH} 5.8 \pm 0.1$ and surface tension $72.5 \mathrm{mN} / \mathrm{m}$ at $22^{\circ} \mathrm{C}$.

Solutions or dispersions of the following surfactants: egg lecithin (Lipoid, Ludwigshafen, Germany), polysorbate 80 (Sigma, St. Louis, USA) or phosphatidylcholine
(Phospholipid, Cologne, Germany) and solutions of preservatives: benzalkonium chloride (50\% solution, $\mathrm{FeF}$ Chemicals A/S, Køge, Denmark), methylparaben (Fluka, Steinheim, Germany) were prepared.

\subsection{Preparation of Formulations}

The composition of the investigated solutions is given in Table 1. Glycerolum has a total concentration of 2.5 $\mathrm{mg} / \mathrm{ml}$ whereas lecithin quantity was chosen to obtain an interface saturated with the phospholipid molecules but to avoid the formation of liposomes in the aqueous phase.

\subsubsection{Monocomponent Solutions}

Lecithin dispersion in water (WLD) containing $2.4 \%$ $\mathrm{w} / \mathrm{w}$ of egg lecithin was prepared at $60^{\circ} \mathrm{C}$, mixing suspension for $1 \mathrm{~h}$ with a magnetic stirrer and for $2 \mathrm{~min}$ with a high shear mixer (8000 rpm), followed by $10 \mathrm{~min}$ sonication $(20 \mathrm{kHz})$. In the meantime $\mathrm{pH}$ was adjusted to 8.0 with $\mathrm{NaOH}$ solution. The resulting WLD was passed through $0.45 \mu \mathrm{m}$ filter (cellulose esters, Millipore) and cooled at room temperature [10]. Before measurement of surface tension, series of WLD dilutions, in the range of lecithin concentration given in Table 1, were prepared by adding water in ratio $1: 1$.

In the same manner aqueous solutions of phosphatedylcholine $(2.4 \% \mathrm{w} / \mathrm{w})$ and polysorbate $80(2.4 \% \mathrm{w} / \mathrm{w})$ were prepared and further diluted. The concentration range of phosphatidylcholine and polysorbate dilutions is collected in Table $\mathbf{1}$.

A solution of methylparaben $(3.60 \mathrm{mg} / \mathrm{g})$ was prepared by dissolving methylparaben in water under constant stir-

Table 1. Mixtures of surfactants with preservatives-formulation data.

\begin{tabular}{|c|c|c|c|}
\hline Surfactant & $\mathrm{c}(\mathrm{mg} / \mathrm{g})$ & Preservative & $\mathrm{c}(\mathrm{mg} / \mathrm{g})$ \\
\hline \multirow{4}{*}{ Egg lecithin } & $4.70 \times 10^{-2}-12.00$ & - & - \\
\hline & $4.70 \times 10^{-2}-12.00$ & Methylparaben & 1.80 \\
\hline & $4.70 \times 10^{-2}-12.00$ & BAC & 0.20 \\
\hline & 12.00 & $\mathrm{BAC}$ & $6.25 \times 10^{-3}-3.40$ \\
\hline \multirow{3}{*}{ Phosphatidylcholine } & $1.17 \times 10^{-2}-12.00$ & - & - \\
\hline & $1.17 \times 10^{-2}-12.00$ & Methylparaben & 1.80 \\
\hline & $1.17 \times 10^{-2}-12.00$ & $\mathrm{BAC}$ & 0.20 \\
\hline \multirow{3}{*}{ Polysorbate 80} & $2.29 \times 10^{-5}-12.00$ & - & - \\
\hline & $2.29 \times 10^{-5}-12.00$ & Methylparaben & 1.80 \\
\hline & $2.29 \times 10^{-5}-12.00$ & BAC & 0.20 \\
\hline
\end{tabular}


ring on a magnetic stirrer for $24 \mathrm{~h}$ at $50^{\circ} \mathrm{C}$. Benzalkonium chloride $(10.0 \mathrm{mg} / \mathrm{g})$ was dissolved in water at room temperature. Series of dilutions in water were prepared within the concentration range given in Table $\mathbf{1}$.

\subsubsection{Surfactant-Preservative Mixtures}

These mixtures contained different concentrations of surfactants and constant concentration of each preservative: benzalkonium chloride $(0.2 \mathrm{mg} / \mathrm{g})$ or methylparaben $(1.8 \mathrm{mg} / \mathrm{g})$. Series of dilutions were performed by sequential dilutions $(1: 1)$ of the surfactant solutions with solutions of benzalkonium chloride $(0.4 \mathrm{mg} / \mathrm{g})$ or methylparaben $(3.6 \mathrm{mg} / \mathrm{g})$, respectively. Additionally, dilutions of benzalkonium chloride containing constant concentration of lecithin $(12.0 \mathrm{mg} / \mathrm{g})$ were prepared (Table 1).

\subsection{Surface Tension Measurement}

Surface tensions were measured with a Wilhelmy plate technique using a piece $(50 \mathrm{~mm} \times 60 \mathrm{~mm} \times 0.25 \mathrm{~mm})$ of filter paper (Whatman No. 1, Madstone, England) attached to the arm of a force sensor (GM2 + UL5, Scaime, France). The laboratory-built set-up was manually operated. A circular $(10 \mathrm{~cm}$ diameter) glass vessel $(1.5 \mathrm{~cm}$ deep) placed on a horizontal support and containing the investigated solution was slowly moved down and the plate detachment force (proportional to the sensor signal voltage) was registered with a digital voltmeter. The measurements were characterized by accuracy within 0.1 $\mathrm{mN} / \mathrm{m}$ and repeatability $\pm 0.3 \mathrm{mN} / \mathrm{m}$. A new Wilhelmy plate was used in each experiment. The measurements were performed at $22^{\circ} \mathrm{C}$, with a sample equilibration time of $30 \mathrm{~min}$. Each concentration probe was tested in triplicate.

\section{RESULTS AND DISCUSSION}

\subsection{Surface Characteristics of Surfactant and Preservative Aqueous Solutions}

The principal parameters of surface characteristics for surfactants and preservatives derived from surface tension vs. concentration plots, shown in Figure 1, are collected in Table 2.

The interpretation of the data in Figure $\mathbf{1}$ is straightforward and conventional. Near the cmc, surfactants begin to form micelles in the bulk aqueous phase. As a result, at or above the cmc a further increase in surfactant monomers gives very little decrease in the surface tension. Until micelles are formed, the value of $\gamma$ reflects the additive molecules dissolved in a bulk aqueous phase.

Figure 1 shows that as the concentration of methylparaben increases, surface tension decreases indicating that the agent is to a certain extent surface active. The surface tension vs. concentration curve does not show any distinct break (no leveling off of $\gamma$ with ln concentration). This result suggests that methylparaben does not form micelles within the concentration range studied [15]. It should be noted that not all surface-active molecules or ions form micelles [16]. This behavior has also been evidenced with some drugs that are shown to be surface active, but do not form micelles [17]. This work highlights that not all surface-active molecules form micelles.

Polysorbate 80 is the nonionic most surface active agent among the studied surfactants $(\mathrm{cmc}=0.1 \mathrm{mg} / \mathrm{g}$, $\left.\gamma_{\mathrm{cmc}}=38.0 \mathrm{mN} / \mathrm{m}\right)$, and $A_{\text {molec }}=224.40 \AA^{2}$ ). Polysorbate 80 is capable of forming aggregates with an aggregation number $\sim 60$ in aqueous solutions at concentrations higher than $0.012 \times 10^{-3} \mathrm{M}$ [18]. Whereas such surfacetants like lecithin or anionic surfactant Aerosol OT (dioctyl sodium sulfosuccinate) form small aggregates (oligomers) with a low aggregation number. In these systems, a smooth discontinuity of the $\gamma(c)$ plot can be first observed at the so-called critical aggregation concentration (cac) then followed by the another one at cmc where regular micellar structures are expected to appear (see phosphatidylcholine at Figure 1). BAC is a cationic surface active agent with $\mathrm{cmc}=0.34 \mathrm{mg} / \mathrm{g}$ and $\gamma_{\mathrm{cmc}}=39.5$ $\mathrm{mN} / \mathrm{m}$. The limiting area per molecule $685.98 \AA^{2}$ is rather large for such a molecule. Methylparaben despite its apparent surface activity does not form micelles. The surface molecular area, achievable for highest concentrations is $118.04 \AA^{2}$.

The slope of $\gamma$ vs. In concentration is proportional to surface excess $(\Gamma)$ as apparent from Eq.1. Table 2 summarizes particular surface characteristics of model surfactant and additive preservatives layers at air/water interface, derived from the plots in Figure 1: cmc, $\gamma_{\mathrm{cmc}}$, surface activity $\sigma$ (obtained from a linear part of the $\gamma(c)$ dependence), and the corresponding $A_{\text {molec }}$ related to $\Gamma$.

Egg lecithin (Lipoid E 80) has $\mathrm{cmc}$ at $0.85 \mathrm{mg} / \mathrm{g}$ and its surface effectiveness (the surface tension reduction at

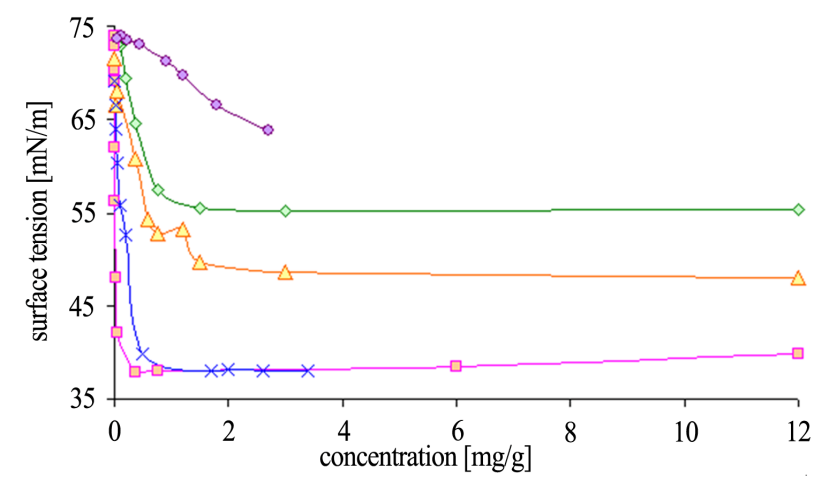

Figure 1. Surface tension vs. concentration plots, for model surfactants and preservatives obtained with a Wilhelmy plate method at $22^{\circ} \mathrm{C}\left(\right.$ methylparaben, $^{\circ}$ egg lecithin, $\triangle$ phosphatydylcholine, $\times$ BAC, $\square$ polysorbate 80 ). 
Table 2. Estimated molecular area $\left(\mathrm{A}_{\text {molec }}\right)$, surface activity $(\sigma)$, surface effectiveness $\left(\gamma_{\mathrm{cmc}}\right)$, and cmc values derived from surface tension vs. In concentration plots, for model surfactant and preservative aqueous solutions. Mean values \pm standard deviation.

\begin{tabular}{lcccc}
\hline Substance & $\mathrm{cmc}(\mathrm{mg} / \mathrm{g})$ & $\gamma_{\mathrm{cmc}}(\mathrm{mN} / \mathrm{m})$ & $\mathrm{A}_{\text {molec }}\left(\AA^{2}\right)$ & $|\sigma|(\mathrm{mN} / \mathrm{m})^{*}$ \\
\hline Basic surfactants & & & & $70,000 \pm 1400$ \\
Polysorbate 80 & $0.100 \pm 0.012$ & $38.0 \pm 2.0$ & $224.4 \pm 11.2$ & $39,282 \pm 785$ \\
Phosphatidylcholine & $0.920 \pm 0.119$ & $50.0 \pm 3.5$ & $90.4 \pm 3.6$ & $22,363 \pm 670$ \\
Egg lecithin & $0.850 \pm 0.102$ & $55.5 \pm 3.3$ & $68.9 \pm 3.5$ & $5000 \pm 150$ \\
Additive surfactants & - & & $(118.0 \pm 7.1)^{1}$ & $48,000 \pm 1440$ \\
Methylparaben & $0.360 \pm 0.039$ & $38.5 \pm 1.9$ & $685.9 \pm 34.3$ & \\
BAC & & &
\end{tabular}

${ }^{1}$ obtained at the highest achievable concentration $(\mathrm{c}=2.7 \mathrm{mg} / \mathrm{g}){ }^{*}$ absolute surface activity in $(\mathrm{mN} / \mathrm{m})$.

the $\mathrm{cmc}$ ) $\gamma_{\mathrm{cmc}}$ is $54.5 \mathrm{mN} / \mathrm{m}$. The limiting $A_{\text {molec }}$ is equal to $68.90 \AA^{2}$. Phosphatidylcholine exhibited a similar value of $\mathrm{cmc}(0.9 \mathrm{mg} / \mathrm{g})$ with higher surface effectiveness $\left(\gamma_{\mathrm{cmc}}\right.$ $=43.0 \mathrm{mN} / \mathrm{m}$, and $A_{\text {molec }}=90.44 \AA^{2}$ ). Surface activity of natural phosphatidylcholines of different orgin was already evaluated but using a different technique i.e., surface balance method [19]. Surface tension lowering of water solution equal to 21.4 and $21.7 \mathrm{mN} / \mathrm{m}$, for soybean phosphatidylcholine and DPPC (dipalmitolphoshatidylcholine), respectively is reported.

These data indicate that the packing area obtained for phosphatidylcholine is about $30 \%$ higher than the area of egg lecithin. Since egg lecithin consists in $84 \%$ of phosphatidylcholine and "impurities", the formation of interfacial molecular complexes or intermolecular interactions with apparently surface-active impurities both contribute to the observed condensing film effect, as already observed in lecithin-cholesterol mixtures [20]. Assuming for lecithin the molecular surface area $72 \AA^{2}$ [21], and the molecular weight $\mathrm{M}=720 \mathrm{~g} / \mathrm{mol}$, of phosphatidylcholine, the surface concentration of a monomolecular layer $\Gamma_{\infty}=\mathrm{M} / \mathrm{N}_{\mathrm{A}} A_{\text {molec }}$ amounts to $1.66 \times 10^{-7} \mathrm{~g} / \mathrm{cm}^{2}$. As indicated above, the area per molecule (i.e. $1 / \Gamma \mathrm{N}_{\mathrm{A}}$ ) was determined to be about $60 \AA^{2}$, for egg lecithin. This value is about twice the size of the cross sectional area occupied by a hydrocarbon chain in a fatty acid, and therefore comparable to the size of a phospholipid such as DPPC (1,2-dipalmitoyl-sn-glycero-3-phosphocholine). To address the observed differences in the limiting molecular area of lecithins derived from different sources, the following $A_{\text {molec }}$ values (in $\AA^{2}$ ) for dipalmitoyl lecithin (51.9), egg yolk lecithin (73.8), soy bean lecithin (78.1), and dioleyol lecithin (87.5) at surface tension of 50 $\mathrm{mN} / \mathrm{m}$ were reported [22]. These results, supplemented with data from analyses of the fatty acid compositions of these lecithins (by gas chromatography), indicate that the degree of unsaturation or the presence of double bonds in fatty acid chains influences the average molecular area of lecithins. Values of $A_{\text {molec }}$ obtained in these comprehendsive studies are comparable for egg lecithin but lower than for phosphatidylcholine [23], as evidenced in our data. The difference may result from the incorporation of surface active impurities such as (phosphatidylethanolamine $8.0 \%$, lysophosphatidylcholine $2.2 \%$, lysophosphatidylethanolamine, sphingomyelin, cholesterol $0.7 \%$, and about $2 \%$ of non-polar triglycerides and free fatty acids) in lecithin.

As shown in Figure 1, the surface tension decreases with increasing lecithin concentration until the saturated monomolecular layer at the particular concentration is formed. Further addition of lecithin does not change the surface tension anymore but would lead to the formation of oligomers in the aqueous phase. As can be seen, the studied substances are capable of lowering the water surface tension, up to its solubility limit, by approximately $20-30 \mathrm{mN} / \mathrm{m}$. It is of interest to compare surface effectiveness $\gamma_{\mathrm{cmc}}$ for a group of model surfactants widely used in physical chemistry studies. In particular, SDS (sodium dodecyl sulfate) lowers the surface tension of water to $\sim 44$, DTAB to $\sim 35$ and $\mathrm{C}_{12} \mathrm{E}_{\mathrm{m}}$ to $\sim 32 \mathrm{mN} / \mathrm{m}$. Low values of surface effectiveness correspond to low surface free energy of the interfacial system which promotes microemulsion formation, increasing its stability and facilitates molecular exchange processes at interfaces.

Among the studied substances the most surface active agents are polysorbate 80 and BAC (lowest cmc and $\gamma_{\mathrm{cmc}}$ while $|\sigma|$ is highest, see Table 2). In addition, high values of $|\sigma|$ in $(\mathrm{mN} / \mathrm{m})$, indicate that the mentioned substance molecules are most hydrophobic and slightly soluble in the bulk as $|\sigma| \sim \Gamma / \mathrm{c}$. Significantly less surface active lecithin and phosphatidylcholine exhibited similar surface signatures. Comparable values of cmc, $\gamma_{\mathrm{cmc}}$, and $|\sigma|$ can be noticed for these similar chemically substances. However, apparent differences between each other can likely result from the presence of impurities in lecithin 
samples (mentioned above) accounting for up to $16 \%$ $\mathrm{w} / \mathrm{w}$. That is confirmed with a local minimum appearing at the $\gamma(c)$, which is usually attributed to the inclusion of surface active components as impurities.

The cmc of ionic surfactants is sensitive to ionic strength of aqueous subphase [24]. A drop in the cmc results in more surfactant molecules going to the bulk of the solution to form micelles rather than going to the surface.

\subsection{Interfacial Signatures of Mixed Surfactant Films}

The shape of the $\gamma$ vs. lecithin concentration curve, for a methylparaben-egg lecithin system, where methylparaben concentration is fixed at $1.8 \mathrm{mg} / \mathrm{g}$, shown in Figure 2(a), is drastically different from that of Figure 1. However, the shape of these plots are reminiscent of those observed when "impurities" are present in surfacetant solutions. One can interpret the observed $\gamma$ vs. lecithin concentration curve in terms of the additive detergent as a deliberately added impurity as follows. The additive preservative (methylparaben) is a more surface active material than lecithin. At the concentrations employed, methylparaben does not form a saturated monolayer on the surface, so that initial addition of lecithin reduces the value of $\gamma$ as added lecithin monomers fill unsaturated sites on the surface. At $\mathrm{c}=0.468 \mathrm{mg} / \mathrm{g}$ of egg lecithin, the value of $\gamma$ ceases to decrease and begins to increase i.e., a local minimum at the $\gamma(c)$ plot is observed. The increasing trend is similar to those found in the systems of binary micelles or those containing impurities [2]. This reversal in the behavior of $\gamma$ is due to the removal of methylparaben molecules from the surface as a result of formation of mixed micelles of methylparaben and egg lecithin in the bulk aqueous phase. At the same time, added lecithin monomers are being deposited on the surface, in competition with formation of co-micelles. At $c_{\text {rem }}=1.321 \mathrm{mg} / \mathrm{g}$ of egg lecithin, a local maximum $\gamma_{\max }$ at the $\gamma(c)$ plot is noticed that corresponds to the nearly complete removal of methylparaben molecules from the surface and subsequently the value of $\gamma$ begins to decrease again as lecithin monomers fill the unsaturated sites remained on the surface. At concentration 12 $\mathrm{mg} / \mathrm{g}$ of lecithin, the value of $\gamma$ approaches the value $\gamma_{\mathrm{cmc}}$ for pure lecithin solution $55 \mathrm{mN} / \mathrm{m}$ (see Table 3, for $c$ above its $\mathrm{cmc}$ ).

We can define three regions and two transitions for the $\gamma$ vs. lecithin concentration plots depicted in Figure 2 (a). Vertical lines point to the concentration border values corresponding to regions from I to III. Critical micellar concentrations are indicated with arrows. Co-ordinates of the local maxima and minima i.e., particular lecithin concentrations as well as their heights $\left(\gamma_{\text {newcmc }}\right)$ can be a measure of surface activity and a partitioning degree, for preservatives characterization in 2-compound compositions. In region I, the value of $\gamma$ decreases with added lecithin concentration and in region II the value of $\gamma$ increases with lecithin concentration. The value of lecithin concentration i.e., mixture $\mathrm{cmc}$ ( new $\mathrm{cmc}$ ), for transition from I to II is smaller for the higher initial concentration of the additive. For the particular methylparaben concentration where almost the saturated monofilm is formed at the surface, the region I is almost absent and $\gamma$ of the surfactant mixture begins to increase starting from the local minimum $\gamma_{\min }$ even for the lowest lecithin concentrations. The transition I $\rightarrow$ II is sharp in the $\gamma$ vs. lecithin concentration curve at higher initial methylparaben concentrations, formation of mixed micelles begins at lower concentrations of lecithin and continues to form until lecithin micelles and lecithin-rich mixed micelles dominate the behavior of the colloid system. This result is consistent with the removal of the additive molecules at the interface because of displacement by lecithin and formation of mixed micelles, since mixed micelles form at lower lecithin concentration as the initial concentration of the preservative is increased. Although, the value of lecithin concentration required for the transition from region I to II depends on the constant initial concentration of the additive. In opposite, the value of lecithin concentration required for the transition from region II to III is independent of the initial additive concentration. These results are consistent with the formation of lecithin micelles becoming the controlling factor in the value of $\gamma$; i.e., region II is one that is rich in lecithin micelles. The transition region II $\rightarrow$ III in the $\gamma$ vs. lecithin concentration curve appears for higher concentrations than the local maximum $\gamma_{\max }$ is observed. This correlates with the completion of the lecithin monomer-micelle phase transition. Further increase in lecithin concentration simply increases the concentration of lecithin micelles. The same $\gamma(c)$ plot is presented in Figure 2 (a), for BAC as an additive. Region I is very broad where surface tension varied only within $3-4 \mathrm{mN} / \mathrm{m}$. For higher concentrations, $\gamma_{\min }$ corresponding transition from region I to II can be hardly distinguished. Finally, the second maximum related to $c_{\text {rem }}$ in region III is almost absent. It should be noticed that in such a 2-component system surface active preservative is of 2 times higher $|\sigma|$ than the surfactant that made it more difficult to remove from the interface by lecithin, as indicated with higher value of $c_{\text {rem }}$ in reference to methylparaben case.

It should be pointed out that for the preservative, which is not as surface-active as methylparaben is, the $\gamma$ vs. lecithin concentration curves could have been of different shape. A rather broad and less deep minimum at $\gamma_{\min }$ could be exhibited. In addition, a maximum $\gamma_{\max }$ corresponding to region II could not appear at all. The 
absence of region II behavior is ascribed to the poorer surfactant ability to form mixed micelles.

The presented model can be also adapted to the reversed mixture situation i.e., surface tension is plotted versus $\mathrm{c}$ of less surface active additive while the concentration of more surface active surfactant is fixed and kept under its cmc. Such a dependence has also the local minimum indicating the mixed micelle formation [25].

An exemplary plot $\gamma(c)$ is shown in Figure 2(b), for a surfactants mixture representing the so-called "inverted system" i.e., a less surface active non-ionic agent (egg lecithin) at fixed $\mathrm{c}=1.2 \mathrm{mg} / \mathrm{g}$ far under its $\mathrm{cmc}$, and ionic preservative (BAC) of higher $\sigma$ with concentration varied up to $4.4 \mathrm{mg} / \mathrm{g}(\sim 12 \mathrm{cmc})$. In region $\mathrm{I}$, egg lecithin forms an unsaturated surface layer at these concentrations. As BAC concentration increases, the accompanying decrease of $\gamma$ is observed since the empty sites at the interface are successively filled with BAC molecules. The adsorption process is finished at $\mathrm{c}=0.100 \mathrm{mg} / \mathrm{g}$, where the local minimum of $\gamma(c)$ occurs equal to 42.6 $\mathrm{mN} / \mathrm{m}$. There is a transition from region I to II which corresponds to the beginning of mixed micelles formation in the bulk phase. It appears that an addition of egg lecithin to the solution of BAC results in a shift of BAC $\mathrm{cmc}$ from 0.6 to $0.10 \mathrm{mg} / \mathrm{g}$. Further increase of BAC concentration leads to the removal of egg lecithin molecules from the interface and formation of mixed micelles.

At $c_{\text {rem }}$ concentration $1.1 \mathrm{mg} / \mathrm{g}$, which corresponds to the local maximum at the surface tension plot, a complete removal of egg lecithin molecules from the interface is completed. For higher BAC concentrations (re- gion III), the interface is progressively saturated with BAC monomers, which is accompanied by a decrease of $\gamma$ up to $38.5 \mathrm{mN} / \mathrm{m}$ ( $\sim \gamma_{\mathrm{cmc}}$, for BAC aqueous solution). The cha- racteristic parameters of the $\gamma(c)$ plot from Figure 2(b) are also collected in Table 3.

The ideal mixing theory is found to adequately describe cmc, monomer concentrations, and micelle composition for the systems where ideal mixing might be expected, i.e., homologous series of surfactants. It however fails to predict $\mathrm{cmc}$ and micelle composition for systems that deviate from ideality such as surfactants with widely differing head groups. The regular solution theory provides a reasonable mathematical approach that was found to be applicable to a variety of anionic, cationic, and nonionic surfactant mixtures almost all of which exhibited negative deviations from ideality.

Phase behavior of cationic/anionic surfactant mixtures strongly depends on the molar ratio and actual concentration of the surfactants [26]. Cationic surfactants have a grater tendency to be incorporated in mixed micellesthan anionic ones. Addition of small amounts of cationic surfactant to the anionic surfactant, near or above its $\mathrm{cmc}$, and vice versa, results in a shift of the cmc of the sur- facetant in excess toward lower concentration. Two main factors are responsible for the lowering of the cmc: an increase in the entropy of mixing of the surfactant with opposite charge and a decrease in the electrical work of micellization due to the decrease of the surface charge density caused by solubilized surfactant of opposite charge [25].

In general, as soon as micelles are formed, the additive molecules are preferentially solubilized in the micelle

Table 3. Surface parameters of 2-component mixed surfactant-preservative (additive) films at aqueous solution at $22^{\circ} \mathrm{C}$. Mean values \pm standard deviation.

\begin{tabular}{|c|c|c|c|c|c|c|c|c|c|}
\hline \multirow{2}{*}{ Surfactant } & \multirow{2}{*}{ Additive } & $\begin{array}{c}\text { Concentration } \\
\text { of additive }\end{array}$ & $\mathrm{cmc}$ & $\gamma_{\mathrm{cmc}}$ & $|\sigma|$ & $c_{\mathrm{rem}}$ & $\gamma_{\mathrm{rem}}$ & $A_{\text {exp }}$ & $\Delta A_{\text {molec }}$ \\
\hline & & $(\mathrm{mg} / \mathrm{g})$ & $(\mathrm{mg} / \mathrm{g})$ & $(\mathrm{mN} / \mathrm{m})$ & $(\mathrm{mN} / \mathrm{m})$ & $(\mathrm{mg} / \mathrm{g})$ & $(\mathrm{mN} / \mathrm{m})$ & $\left(\AA^{2}\right)$ & $\left(\AA^{2}\right)$ \\
\hline \multirow[t]{3}{*}{ Polysorbate 80} & Methylparaben & 1.8 & $0.046 \pm 0.002$ & $51.5 \pm 2.1$ & $16,823 \pm 672$ & $0.150 \pm 0.020$ & $52.8 \pm 2.1$ & $636.5 \pm 25.4$ & $425.82 \pm 12.7$ \\
\hline & $\mathrm{BAC}$ & 0.2 & $0.023 \pm 0.001$ & $46.9 \pm 1.9$ & $30,043 \pm 901$ & $0.093 \pm 0.003$ & $47.6 \pm 1.4$ & $801.3 \pm 32.0$ & $573.50 \pm 17.2$ \\
\hline & - & - & $0.100 \pm 0.012$ & $38.0 \pm 2.0$ & & & & & \\
\hline \multirow[t]{3}{*}{ Egg lecithin } & Methylparaben & 1.8 & $0.468 \pm 0.014$ & $54.3 \pm 2.2$ & $35,752 \pm 980$ & $1.321 \pm 0.039$ & $55.0 \pm 1.7$ & $68.1 \pm 2.7$ & $-7.2 \pm 0.2$ \\
\hline & $\mathrm{BAC}$ & 0.2 & $0.350 \pm 0.010$ & $52.9 \pm 2.1$ & $48,901 \pm 978$ & $1.537 \pm 0.046$ & $54.5 \pm 1.6$ & $53.3 \pm 1.6$ & $-125.5 \pm 3.7$ \\
\hline & - & - & $0.850 \pm 0.030$ & $55.5 \pm 3.3$ & & & & & \\
\hline \multirow[t]{3}{*}{ Phosphatidylcholine } & Methylparaben & 1.8 & $0.387 \pm 0.011$ & $34.5 \pm 1.4$ & $15,004 \pm 300$ & $0.850 \pm 0.030$ & $39.5 \pm 1.2$ & $120.9 \pm 3.6$ & $26.8 \pm 0.8$ \\
\hline & $\mathrm{BAC}$ & 0.2 & $0.210 \pm 0.010$ & $56.5 \pm 2.3$ & $45,192 \pm 903$ & $0.951 \pm 0.032$ & $52.3 \pm 1.6$ & $132.4 \pm 5.2$ & $29.8 \pm 0.9$ \\
\hline & - & - & $0.900 \pm 0.035$ & $50.0 \pm 3.5$ & & & & & \\
\hline \multicolumn{10}{|c|}{ Inverted system } \\
\hline \multirow[t]{2}{*}{$\mathrm{BAC}$} & Egg lecithin & 12.0 & $0.100 \pm 0.010$ & $42.6 \pm 1.7$ & $22,363 \pm 670$ & $1.101 \pm 0.032$ & $53.8 \pm 1.6$ & & \\
\hline & - & - & $0.360 \pm 0.020$ & $38.5 \pm 1.9$ & & & & & \\
\hline
\end{tabular}




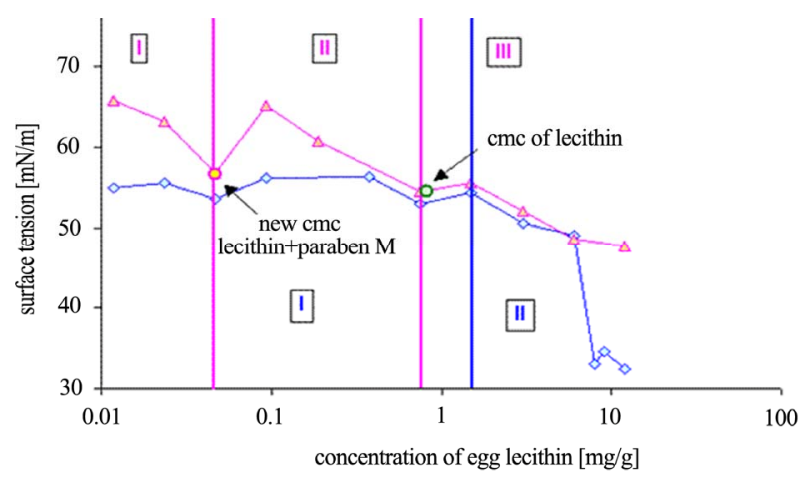

(a)

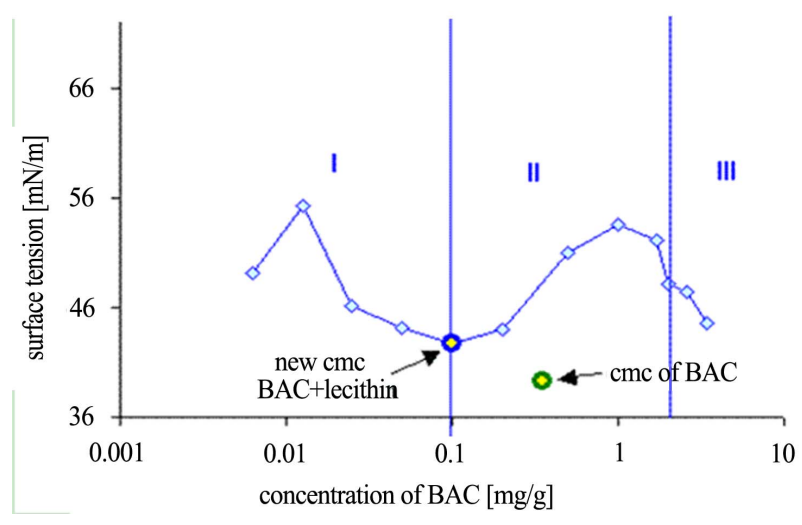

(b)

Figure 2. (a) Surface tension vs. In concentration of egg lecithin, for: $\Delta$ methylparaben-egg lecithin system and $\bullet$ BACegg lecithin system with BAC at $22^{\circ} \mathrm{C}$; (b) Surface tension vs. ln concentration of BAC, for so-called an "inverted system".

aggregates, and are mostly removed from the aqueous phase. Because the micelles provide a less polar environment for the additive molecules, they are solubilized in micelles since the process is energetically favored.

The following general tendency of the parameters variability can be noticed: cmc $\downarrow, \gamma_{\text {cmc }} \downarrow$ with an increase of surface activity $(|\sigma| \uparrow)$ of the additive preservative.

Since a surfactant-preservative system is composed of two surfactants, surface competitive adsorption takes place. For mixtures, relative activities favor enrichment of certain components i.e., the preferential desorption of hydrophilic components can leave behind the more hyophobic components (of higher $|\sigma|)$ ). In particular, methylparaben has $|\sigma|$ value about 10 times lower than BAC and is better soluble in the aqueous phase in a form of free molecules being more available for the contact with microorganisms.

Deviations from the ideal mixing behavior for each surfactants pair of the studied surfactant mixture can be expressed by the difference of limiting molecular areas $\Delta A_{\text {molec }}=A_{\text {exp }}-A_{12}$ derived from the experimental $\gamma(c)$ plots and theoretical dependence (Eq.4). For formulations of polysorbate 80 and phosphatidylcholine with additive systems, positive deviations (from 527.5 to 425.8) and (from 29.8 to 26.8) $\AA^{2}$, with BAC and methylparaben as preservatives, respectively demonstrated repulsive forces between the surfactant molecules at interfaces, which are much stronger for polysorbate 80 being the most surface active surfactant. Positive values of the areas difference for methylparaben additive are always lower than for BAC, and the former molecule requires a lower energy to be removed from the interface to the adjacent underlying bulk phase. Attractive forces were evidenced between surfactant molecules for egg lecithin-additive systems (negative deviations from -7.19 to $-25.48 \AA^{2}$, for BAC and methylparaben, respectively). Again the forces are weaker for the methylparaben-surfactant interaction and such an additive is to be eliminated from the interface easier. For the phosphatidylcholine-preservative system, the presence of surface active impurities in the mixed film structure significantly affects the sign and strength of intermolecular forces.

Surface activity of the additive in the surfactant mixture (apart from its absolute value obtained earlier for a pure component solution and given in Table 2) can be derived according to Eq.2 applied to the $\gamma$ vs. surfactant concentration plot, where the surfactant concentration is fixed while the preservative concentration is varied. The averaged mean values of $|\sigma|$ for the studied preservative-surfactant systems are collected in Table 3 According to Eq.2, the molecular partitioning coefficient of surfactant molecules between the surface and bulk phases $\mathrm{K}_{\mathrm{p}}=\Gamma / \mathrm{c}$ is proportional to $\sigma$. Far higher values of $\sigma$ by an order of magnitude (for the mixtures with the most surface active surfactant polysorbate 80 ) were obtained for BAC in reference to methylparaben that corresponds to its less solubility reflected in the high $\Gamma / \mathrm{c}$ ratio. As a result, methylparaben molecules are more easily removed from the interface and incorporated in mixed micelles formed in the bulk within the applied concentration range of applied surfactants. Such an additive or similar in its surface properties is the most suitable and efficient agent for bacteria destruction in aqueous drug mixtures. However, the concentration of the preservative monomer form in the solution bulk remains to be determined for a particular system by means of the moles conservation law (Eq.3).

Previously, estimated that half of the lecithin may be present in the interface while half is located in the liposomal or micellar structures in the continuous aqueous phase [27]. It is to note, a high content of parabens in the interface region, where depending on the formulation, $38 \%-58 \%$ of the total content of added preservatives is located [1]. Consequently, the low concentration of free parabens in water does not allow to achieve a required antimicrobial protection as demonstrated by a pharmacopoeial test [1]. 


\subsection{Surface Parameters versus Functionality of Additive Preservative in a Surfactant-Preservative Mixture}

In order to quantify the additive preservative effect alone on surface characteristics of the mixed drug-preservative system, the surface parameters can be normalized to the values taken where the preservative surfactant is absent, using data already collected in Tables $\mathbf{2}$ and $\mathbf{3 .}$

At the particular surfactant concentration $c_{\text {rem }}$ (see Figure 2(a)), additive preservative molecules are cometely removed from the interface to form mixed micelles in the bulk. Values of $c_{\text {rem }}$ normalized to the mixture $\mathrm{cmc}$ $\left(\mathrm{cmc}_{\text {new }}\right)$ were as follows: $\mathrm{c}_{\mathrm{rem}} / \mathrm{cmc}_{\text {new }}=3.2$ (polysorbate $80+$ methylparaben); 3.9 (polysorbate $80+$ BAC); 2.8 (egg lecithin + methylparaben); 4.4 (egg lecithin + BAC); 2.1 (phosphatidylcholine + methylparaben); 4.5 (phosphatidylcholine + BAC). Values of the normalized $c_{\text {rem }}$ were comparable, despite the surfactant surface characteristics used, but apparently lower $(2.1$ - 3.2) in reference to $(3.9-4.5)$, for methylparaben and BAC as preservatives, respectively. It seems that $c_{\text {rem }}$ was mainly attributed to surface activity of the additive (preservative) in the solution collected in Table 3. It should be pointed out that surface activity of a surfactant in the 2-compound mixture is comparable to its absolute surface activeity of a pure component in aqueous solution (compare data for $\sigma$ from Table 2) but only for the most surface active preservative studied here (BAC). Whereas, inter-molecular interactions between surfactant and preservative molecules at the interface may increase the "effecttive" surface activity by a factor of $3-7$ (compare $\sigma$ data for methylparaben) depending on the constant initial additive concentration. It is evident that the characteristic concentrations derived from the generalized $\gamma(\mathrm{c})$ plot appeared in a sequence: $\mathrm{cmc}_{\text {new }}<\mathrm{cmc}$ (principal surfactant $)<c_{\text {rem }}$ depended on surface activity of the additive (preservative). Surface tension of the mixed system $\gamma_{\text {rem }}\left(\right.$ at $\left.\mathrm{c}=c_{\text {rem }}\right)$ is close to the value of $\gamma_{\text {cmc }}$ (of surfactant aqueous solutions at their $\mathrm{cmc}$ ).

For an inverted mixture surfactants system i.e., egg lecithin concentration fixed at $12.0 \mathrm{mg} / \mathrm{g}$ and BAC concentration varied, the normalized $c_{\text {rem }}=11$ was much higher while the cmc shift factor remained comparable (3.6), as evidenced for the regular composition plot. In such a case, a shape of the curve exhibits a less deep local minimum (at $\mathrm{cmc}_{\text {new }}$ ) with $\gamma_{\mathrm{cmc}}$ much higher without a pronounced maximum at $c_{\text {rem }}$, observed for preservatives of lower surface activity.

Since the surface signatures of surfactant solutions depend on other factors, the effect of temperature and subphase $\mathrm{pH}$ on surface signatures of surfactant-preservative formulations remains to be addressed in further detailed studies [28].

\section{CONCLUSIONS}

To sum up, surface tension vs. concentration plots obtained for model 2-component surfactant/preservative aqueous solutions allowed: the partitioning of additive surfactant molecules between the surface and bulk phases, interfacial preservative molecules removal and mixed micelles formation conditions to be evaluated. In particular, cmc lowering, surface effectiveness $\gamma_{\mathrm{cmc}}$, and $c_{\mathrm{rem}}$ appeared to be unequivocally related to surface activity $\sigma$, and further to the partitioning coefficient $\Gamma / \mathrm{c}$ of the additive surfactant. A surface tension methodology could be a useful and supplementary tool, apart from chemical analyses, for optimal selection of additive surfactants playing a bacteria destruction role in aqueous drug-preservative systems.

The illustrative results clearly pointed to methylparaben as the most suitable preservative agent since its surface activity is almost 10 times lower than that of BAC. Consequently, as a result the partitioning coefficient is lowered by the same factor, less surface energy and lower $c_{\text {rem }}$ are required to form mixed micelles in the bulk phase. That remains in agreement with the previous work based on chemical analyses of preservatives in submicron lecithin formulations. The concept requires to be tested on a large number of differentiated in their surface properties pairs of surfactant-preservative systems.

It should be pointed out that the apparent values of the interfacial parameters are sensitive to impurities likely to present in the studied samples. Thus, the relative or normalized surface parameters can be useful as indicators of the preservative effectiveness in antimicrobial drug protection characterization.

\section{ACKNOWLEDGEMENTS}

The study was supported by grants: MNiSzW nr 2P05F 04030 and NCN nr N N405 668440, and carried out in part within the framework of the scientific activity of the University of Gdansk (supported from DS/5200-4-0024-12).

\section{REFERENCES}

[1] Sznitowska, M., Janicki, S., Dabrowska, E. and Gajewska, M. (2002) Screening of antimicrobial agents as potential preservatives for submicron emulsions. European Journal of Pharmaceutical Science, 15, 489-495. doi:10.1016/S0928-0987(02)00034-9

[2] Turro, N.J., Kuo, P-L., Somasundaran, P. and Wong, K. (1986) Surface and bulk interactions of ionic and nonionic surfactants. Journal of Physical Chemistry, 90, 288-291. doi: $10.1021 / \mathrm{j} 100274 \mathrm{a} 017$

[3] Watrobska-Swietlikowska, D. and Sznitowska, M. (2006) Partitioning of parabens between phases of submicron emulsions stabilized with egg lecithin. International Journal of Pharmacy, 312, 174-178. 
doi:10.1016/j.ijpharm.2006.01.005

[4] Adamson, A.W. and Gast, A.P. (1997) Physical Chemistry of Surfaces, 6th ed. John Wiley and Sons, New York. doi:10.1149/1.2133374

[5] Schwarz, G., Wackenbauer, G. and Taylor, S.E. (1996) Partitioning of a nearly insoluble lipid monolayer into its aqueous subphase. Colloids and Surfaces A: Physicochemical and Engineering Aspects, 111, 4341-4346. doi:10.1016/0927-7757(95)03495-1

[6] Gaines, G.L. (1966) Insoluble Monolayers at the Liquid/Gas Interface, Wiley, New York.

[7] Sanchez-Gonzales, J., Cabrerizo-Vilchez, M.A. and GalvezRuiz, M.J. (1999) Evaluation of the interactions between lipids and $\gamma$-globulin protein at the air-liquid interface. Colloids and Surfaces B: Biointerfaces, 12, 123-138. doi:10.1016/S0927-7765(98)00069-1

[8] Nguyen, C.M., Rathman, J.F. and Scamehorn, J.F. (1986) Thermodynamics of mixed micelle formation. Journal of Colloid and Interface Science, 112, 438-446. doi:10.1016/0021-9797(86)90112-8

[9] Dharmawardana, U.R., Christian, S.D., Tucker, E.E., Taylor, R.W. and Scamehorn, J.F. (1993) A surface tension method for determining binding constants for cyclodextrin inclusion complexes of ionic surfactants. Langmuir, $\mathbf{9}$, 2258-2263. doi:10.1021/la00033a003

[10] Sznitowska, M., Klunder, M. and Placzek, M. (2008) Paclitaxel solubility in aqueous dispersions and mixed micellar solutions of lecithin. Chemical and Pharmaceutical Bulletin, 56, 70-74. doi:10.1248/cpb.56.70

[11] Polozova, A.I., Dubachev, G.E., Simonova, T.N. and Barsukov, L. (1995) Temperature-induced micellar-lamellar transformation in binary mixtures of saturated phosphatidylcholines with sodium cholate. FEBS Letters, 358, 17-22. doi:10.1016/0014-5793(94)01378-E

[12] Stoye, I., Schröder, K. and Müller-Goymann, C. (1998) Transformation of a liposomal dispersion containing ibuprofen lysinate and phospholipids into mixed micellesphysicochemical characterization and influence on drug permeation through excised human stratum corneum. European Journal of Pharmacy and Biopharmacy, 46, 191-200. doi:10.1016/S0939-6411(98)00023-X

[13] Westesen, K. and Wehler, T. (1992) Physicochemical characterization of a model intravenous oil-in-water emulsion. Journal of Pharmaceutical Science, 81, 777-786. doi:10.1002/jps.2600810812

[14] Herman, C.J. and Groves, M.J. (1993) The influence of free fatty acid formation on the $\mathrm{pH}$ of phospholipid-stabilized triglyceride emulsions. Pharmaceutical Research, 10, 774-775. doi:10.1023/A:1018932421357

[15] Al-Maaieh, A. and Aburub, A. (2007) Surface activity of non-micelle forming compound containing a surface-active impurity. International Journal of Pharmacy, 334, 125-128. doi:10.1016/j.ijpharm.2006.10.034

[16] Mukerjee, P.J. (1974) Micellar properties of drugs: Micellar and nonmicellar patterns of self-association of hydrophobic solutes of different molecular structures-monomer fraction, availability, and misuses of micellar hypothesis. Journal of Pharmaceutical Science, 63, 972-981.

\section{doi:10.1002/jps.2600630647}

[17] King, S.Y., Basista, A.M. and Torosian, G. (1989) Selfassociation and solubility behaviors of a novel anticancer agent, Brequinar Sodium. Journal of Pharmaceutical Science, 78, 95-100. doi:10.1002/jps.2600780204

[18] Hinze, W.L. (1987) Organized surfactant assemblies in separation science. In: Hinze, W.L. and Armstrong, D.W., Eds., Ordered Media in Chemical Separations, ACS Symposium Series No. 342, American Chemical Society, Chapter 1, 2-82. doi:10.1021/bk-1987-0342.ch001

[19] Zakharova, T.S., Fortinskaya, E.S., Kochetova, M.M., Torkhovskaya, T.I. and Khalilov, E.M. (2000) Comparison of distribution characteristics of phosphatidylcholines and surfactant preparation. Biophysics and Biochemistry, 129, 411-413. doi:10.1007/BF02439265

[20] Tajima, K. and Gershfeld, N.L. (1978) Equilibrium studies of lecithin-cholesterol interactions. II. Phase relations in surface films: Analysis of the "condensing" effect of cholesterol. Biophysical Journal, 22, 489-500. doi:10.1016/S0006-3495(78)85501-5

[21] Krickau, D.P., Mueller, R.H. and Thomsen, J. (2007) Degradation kinetics of hydrolytically susceptible drugs in $\mathrm{O} / \mathrm{W}$ emulsions - Effects of interfacial area and lecithin. International Journal of Pharmacy, 342, 62-71. doi:10.1016/j.ijpharm.2007.04.033

[22] Kjelleberg, S. and Stenstrom, T.A. (1980) Lipid surface films: Interaction of bacteria with free fatty acids and phospholipids at the air/water interface. Journal of General Microbiology, 116, 417-423. doi:10.1099/00221287-116-2-417

[23] Vaknin, D., Kjaer, K., Als-Nielsen, J. and Lősche, M. (1991) Structural properties of phosphatidylcholine in a monolayer at the air/water interface. Biophysical Journal, 59, 1325-1332. doi:10.1016/S0006-3495(91)82347-5

[24] Yalkowsky, S.H. (1999) Solubility and Solubilization in Aqueous Media. Oxford University Press, Oxford.

[25] Dragcevic, D., Bujan, M., Grahek, Z. and FilipovicVincekovic, N. (1995) Adsorption at the air/water interface in dodecylammonium chloride/sodium dodecyl sulfate mixtures. Colloid and Polymer Science, 273, 967-973. doi:10.1007/BF00660375

[26] Filipovic-Vincekovic, N., Bujan, M., Dragcevic, D. and Nekic, N. (1995) Phase behavior in mixtures of cationic and anionic surfactants in aqueous solution. Colloid and Polymer Science, 273, 182-188. doi:10.1007/BF00654016

[27] Han, J. and Washington, C. (2005) Partition of antimicrobial additives in an intravenous emulsion and their effect on emulsion physical stability. International Journal of Pharmacy, 288, 263-271. doi:10.1016/j.ijpharm.2004.10.002

[28] Galvez-Ruiz, M.J. and Cabrerizo-Vilchez, M.A. (1992) Mixed monolayers of lecithin and bile acids at the airaqueous solution interface. In: Stroeve, P. and Blalazs, A.C., Eds., Macromolecular Assemblies in Polymeric Systems, ACS Symposium Series No. 493, American Chemical Society, Chapter 13, 135-152. doi:10.1021/bk-1992-0493.ch013 